\title{
Study of the Influence of a Light Source on the Result of the Reconstruction of the Flaccid Membrane of an Artificial Heart
}

\author{
Krzysztof Murawski \\ Military University of Technology \\ Urbanowicza Str. 2, \\ 00-908 Warsaw, Poland, \\ IEEE Member \# 92707852 \\ Email: krzysztof.murawski@wat.edu.pl
}

\author{
Wojciech Sulej \\ Military University of Technology \\ Urbanowicza Str. 2, \\ 00-908 Warsaw, Poland, \\ Email: wojciech.sulej@wat.edu.pl
}

\begin{abstract}
The article presents the influence of the used light sources (illuminators) on the result of the 3D reconstruction of the flaccid membrane surface shape. The research was carried out using a flaccid membrane developed by the authors for the pulsating, extracorporeal, pneumatic heart support pump model. Subsequently, the tested illuminators are an integral part of the sensor for measuring the stroke volume (SV) and the cardiac output (CO). Due to the operation principle of the computer video measurement method $\mathrm{SV}$ and $\mathrm{CO}$ developed by authors, the consistency of the obtained reconstruction of the flaccid membrane surface shape with the actual (reference) shape of the membrane is fundamentally important. For this reason, the influence of the developed lighting constructions was experimentally verified. For this purpose, a laboratory station and a rigid equivalent of the flaccid membrane were created, thus ensuring the reproducibility of the experiments. For LED-based illuminators, printed circuit boards were also designed, which eliminated the impact of changes in the position of light sources. Thanks to this, it was possible to determine the actual impact of the illuminator's construction on the obtained reconstruction result. The tests were performed for several selected light sources. The following were taken into account: LED diodes operating in the visible band, near infrared LED diodes, optical fibers and electroluminescent film.
\end{abstract}

\section{INTRODUCTION}

$\mathrm{T}$ he publication is the result of studying the effect of lighting on the result of the $3 \mathrm{D}$ reconstruction of the flaccid membrane surface shape. Reconstruction of the surface shape was carried out with a proprietary video sensor used to measure the instantaneous stroke volume (SV) and the cardiac output (CO) of the pulsating, extracorporeal, pneumatic myocardial support pump (ventricular assist devices - VAD), described in works [1-5]. One of the known VAD constructions is a heart pump made as part of the "Polish Artificial Heart" program, discussed in [6] and shown in Fig. 4. Based on its description, the first proprietary VAD model shown in Fig. 1 (currently no longer used) has been produced. The research discussed in this paper was carried out using the new pump models shown in Fig. 2 and Fig. 3. They represent modification made by the authors of the original VAD design. The model shown in

This work was not supported by any organization
Fig. 2 was made using a spatial printing technique. The pump shown in Fig. 3 was made using a casting technique. The presented models, except for the blood chamber, have a pneumatic chamber, which view is shown in Fig. 6. The chambers are separated by a non-elastic, flaccid membrane composite visible in Fig. 5 and Fig. 7. The up/down movement of membrane causes the blood to be pushed or sucked to/from the blood chamber. The membrane, along with the markers on it, is illuminated by the illuminator. At the same time, it is observed by the sensor camera. Reconstruction of the surface shape of the membrane, Fig. 2 and Fig. 3, necessary to determine the $\mathrm{SV}$ and $\mathrm{CO}$ is obtained by the visual technique discussed in [5, $7-9]$. This technique uses the distance measurement method described in items [10 - 14].

In works [15-18], it was shown that by stabilizing the level of lighting the markers, it is possible to determine the distance from the markers to the image sensor of the camera and to determine the flaccid membrane surface shape.

\section{MOTIVATION}

The motivation to undertake works aimed at determining the effect of lighting on the result of the reconstruction of the VAD flaccid membrane surface shape was to obtain, as a result of the $3 \mathrm{D}$ reconstruction, the shape of the membrane surface in accordance with the actual state. The consistency with the actual state of the determined shape of the flaccid membrane surface is extremely important due to the

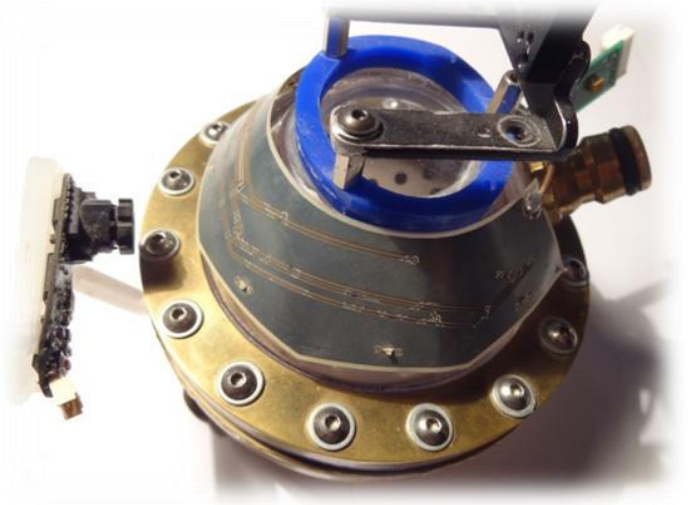

Fig. 1 The first of the pump heart assist models, developed by the authors of this work, based on commercial descriptions of pumps 


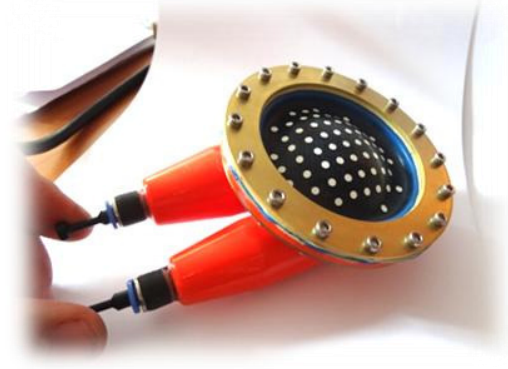

Fig. 2 Currently used proprietary ventricular assist pump model created using 3D printing (2017)

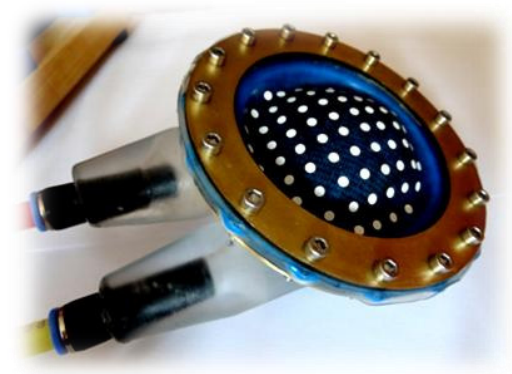

Fig. 3 Currently used proprietary ventricular assist pump model created using a casting technique (2018)

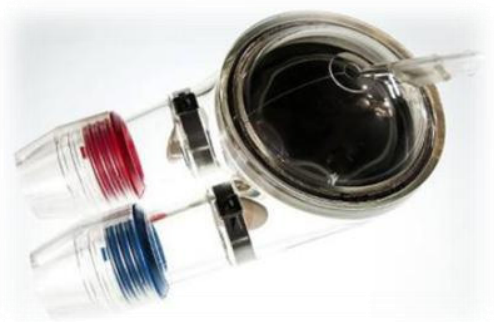

Fig. 4 The extracorporeal pneumatic heart assist pump developed in the framework of the Polish Artificial Heart

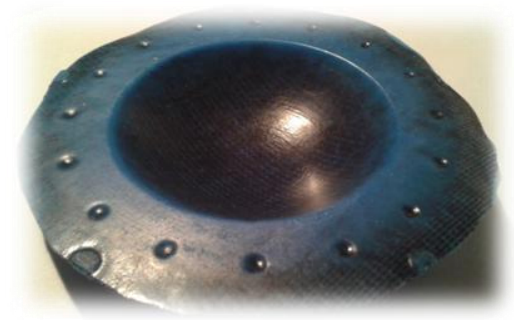

Fig. 5 Proprietary composite flaccid membrane created for pneumatic heart assist pumps shown on Fig. 2 and Fig. 3 (2017)

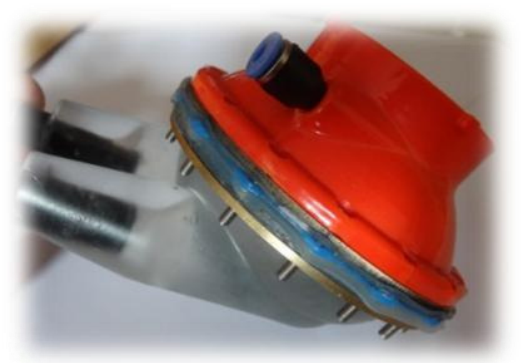

Fig. 6 Blood chamber presented in Fig. 3 including the red pneumatic chamber (2017) precision of determining the VAD's instantaneous stroke volume and the cardiac output. Therefore, it was advisable to examine the influence of the lighting parameters on the accuracy of the determined reconstruction of the flaccid membrane surface shape.

\section{PROBLEM DEFINITION}

The fundamental problem that is being solved in this work is to compensate for the effect of the membrane lighting on the result of $3 \mathrm{D}$ reconstruction of the shape of its surface. This impact is the result of constant changes in the position of the surface of the flaccid membrane in relation to the elements that illuminate it.

The magnitude of the influence of lighting on the result of the reconstruction of the flaccid membrane surface shape depends on: a) the shape of the pneumatic chamber; b) the type of light source and its parameters; c) the mode of light distribution in the pneumatic chamber.

Despite many difficulties, this problem was effectively eliminated in the case of the pump shown in Fig. 1. The following was used: a) an illuminator built out of VSMY 1850X0 diodes arranged in a specially selected pattern; b) PCA 9622DRT controller; c) a visible light blocking filter mounted on the camera lens; d) PMMA material for making a pneumatic chamber. This ensured almost uniform lighting in the entire membrane operation range. Unfortunately, this method in the pumps currently used cannot be implemented, due to their construction, and the small distance of the membrane to the walls of the pneumatic chamber, Fig. 7. For this reason, work was undertaken to develop and study the new lighting constructions.

\section{FITNESS FUNCTION}

An important element of the conducted research was the creation of reference shapes of the flaccid membrane surface, consistent with the actual state. The compatibility study was carried out using the membrane surface shapes defined mathematically, Fig. 8a. These include: a) extremely convex - this is the condition of the membrane, in which the membrane is lifted upwards at its maximum; b) extremely concave - this is the state of the membrane in which the downward membrane is at its maximum; c) flat membrane -

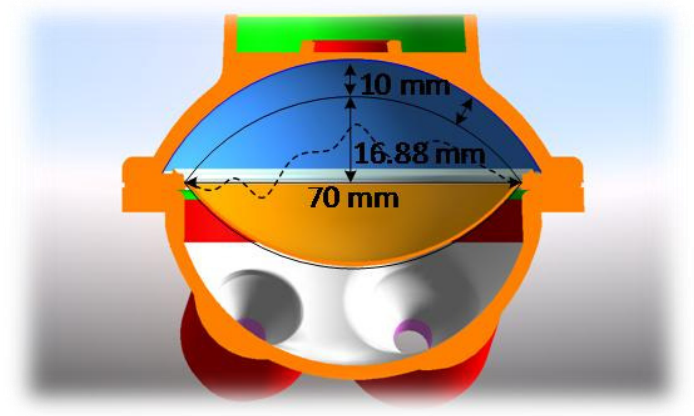

Fig. 7 Cross section of pump model presented in Fig. 6 along with selected dimensions 
a)

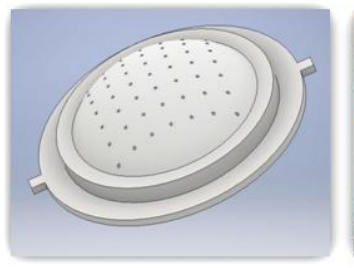

b)
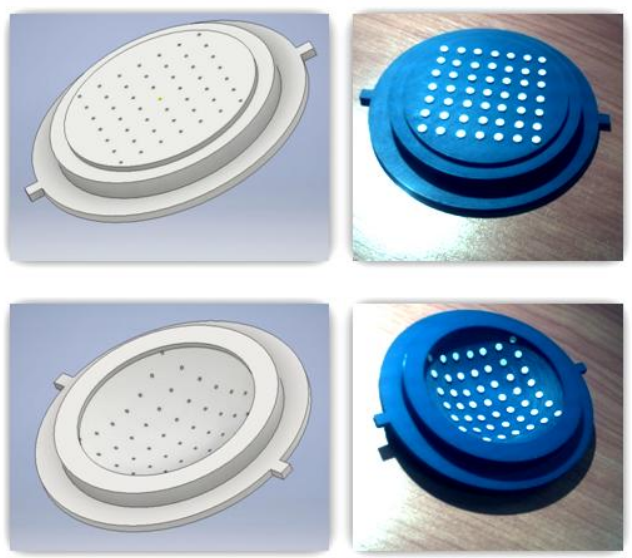

Fig. 8 Designed (a) and produced (b) model membrane shapes in 3D printing technology

a)

b)

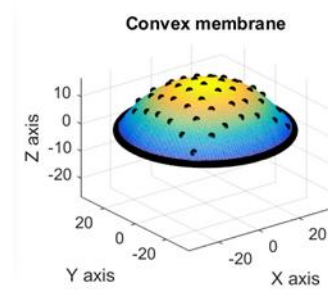

Flat membrane

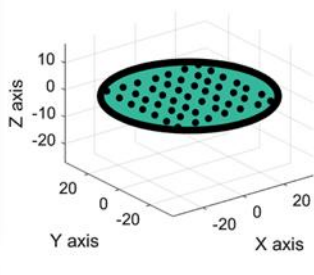

Concave membrane
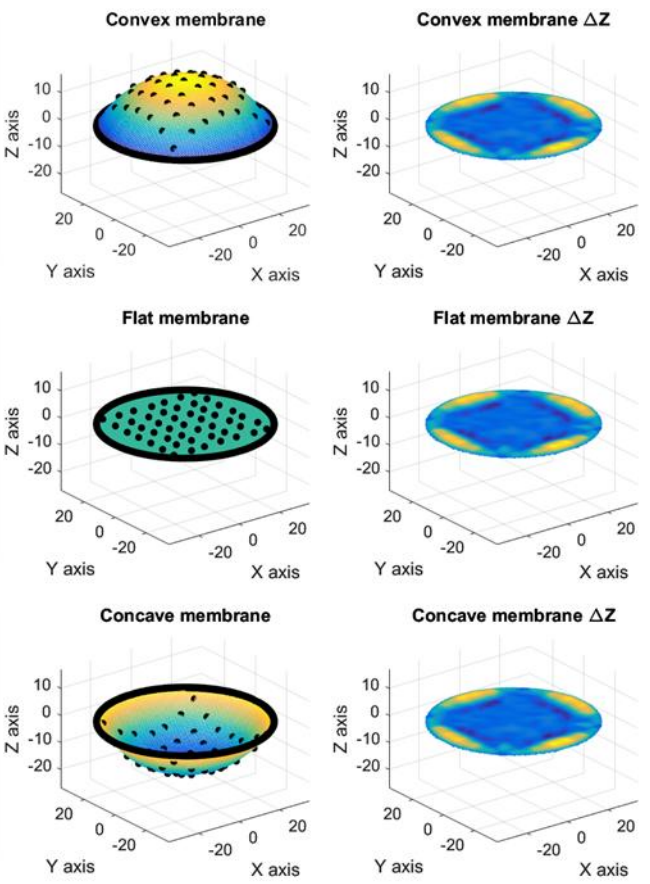

Fig. 9 Model grids determined for membranes from Fig. 8b (a), exemplary charts of membrane reconstruction errors (b)

this is the state in which the entire surface of the membrane is parallel to the plane of the camera image sensor. Due to "additional" material, this condition is not achievable by the membrane shown in Fig. 5. Nevertheless, this membrane is helpful when determining the error of the shape of the membrane surface when it is at the connection level of the blood chamber with the pneumatic chamber.

The reference membranes were modeled in CAD software. As a result, the three-dimensional models shown in Fig. 8a were obtained. These models were then saved in STL format and printed on a 3D printer. The membranes obtained in the manner presented are shown in Fig. 8b. The print was made with a layer height in the $\mathrm{Z}$ axis equal to $0.09 \mathrm{~mm}$. The next step was to create reference grids describing the shape of the tested membranes. These grids were determined from known mathematical descriptions. The obtained reference grids are shown in Fig. 9a. Figure 9b shows sample error diagrams of reconstructing the shape of the membrane surface. They were obtained by determining the position difference in the $\mathrm{Z}$ axis for all the corresponding points on the reference grid and the measurement grid, representing the currently obtained reconstruction result of the membrane surface shape. The differences determined in this way were used to calculate the mean square error (MSE), which for the best solutions was only $0.0041 \mathrm{~mm}^{2}$.

\section{LIGHT SOURCE DESIGN}

The tested illuminator constructions, (A) Fig. 10 and (B), (C) Fig. 11, were made based on the previously tested PCA 9622 DRT system, the application scheme of which is given in Fig. 16 in the paper [20]. 16 PWM channels were used in the illuminators. Each channel controls a LED, which is protected by a resistor limiting the current. The FYLS-0805UWC diode was used in the illuminator (A), working in the visible band at an angle of illumination $\varphi=130 \mathrm{deg}$ and a current $\mathrm{I}_{\mathrm{F}}=20 \mathrm{~mA}$. In systems (B) and (C), infrared diodes were soldered: VSMY 1850X01 $\left(\varphi=120 \mathrm{deg}, \mathrm{I}_{\mathrm{F}}=100 \mathrm{~mA}\right)$ and VSMG $10850(\varphi=150 \mathrm{deg}$, $\mathrm{I}_{\mathrm{F}}=65 \mathrm{~mA}$ ). A high intensity LED OF-LED1G4W is used in the fiber optic illuminant (D) shown in Fig. 10, which introduces light into the fiber optic light shining on the side FOSS-3 [21]. The last tested illuminator (E) was made of EL
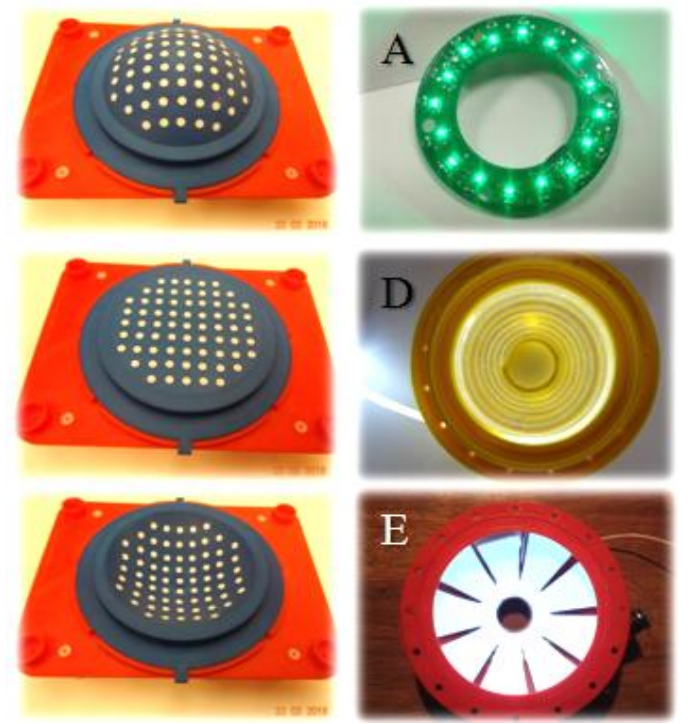

Fig. 10 View of stations and membranes for testing the illuminators (left), view of tested illuminators (right) 


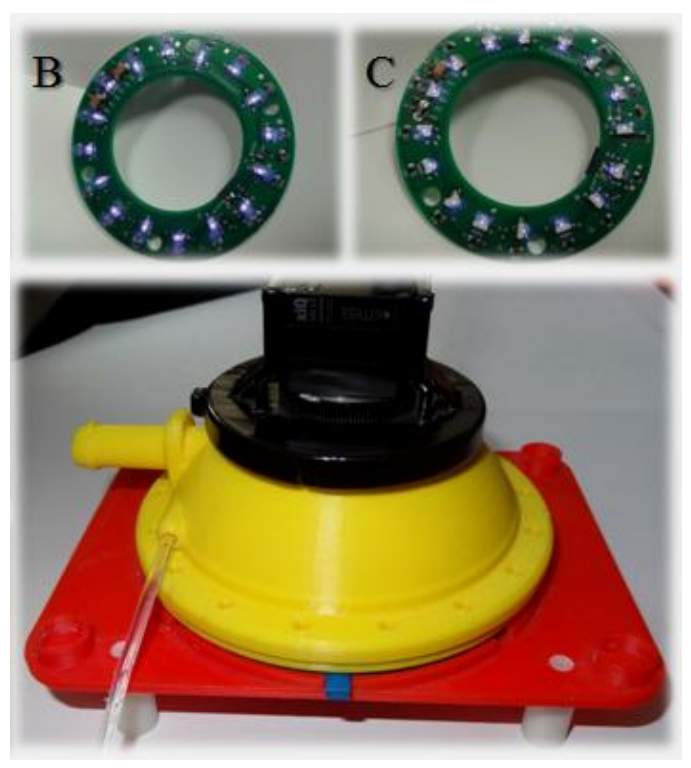

Fig. 11 View of tested illuminators (top), measuring station with a mounted fiber optic illuminator (bottom)

(electroluminescent) film. The EL film was cut into the shape shown in Fig. $10(\mathrm{E})$. The film was powered by the converter, which converts $12 \mathrm{~V}$ DC into AC with an amplitude of $330 \mathrm{~V}$ and a frequency of $1.7 \mathrm{kHz}$. A white EL film was used in the study. Application schematics of specialized converters for powering electroluminescent film can be found in [22].

\section{DESCRIPTION OF THE EXPERIMENT}

The experiments were carried out on a proprietary measuring station, made using 3D printing technology, Fig. 10 (left). Fig. 11 (bottom) presents the pneumatic chamber during testing with the optical fiber illuminator (D) shown in Fig. 10. The testing of illuminators $(A)-(C)$ shown in Fig. 10 and Fig. 11 started by determining the diode current, which was unchanged for all tested membranes. The experiment involving system (A) gave a negative result - the flat and concave membrane (Fig. 12-2a and Fig. 12-3a) were underexposed. Similar effects were obtained for system (B). Although the flat membrane was properly illuminated, the convex membrane was clearly overexposed and the concave membrane underexposed Fig. 12-1b and Fig. 12-3b. Incorrect results were also obtained for system (C). This system uses infrared LEDs shining to the sides. The applied diodes reduced the spots observed on the surface of the convex membrane, but this adversely affected the illumination of the other two membranes, where most of the markers were below the limit of being distinguishable, Fig. 12-2c and Fig. 12-3c. System (D) turned out to be a promising solution. The fiber optic side lighting illuminated the flat and concave membranes well, Fig. 12-2d and Fig. 12-3d, however, the convex membrane remained underexposed, Fig. 12-1d. The reason for this was seen in the significant (approx. $16 \mathrm{~mm}$ ) width of the camera lens, and consequently in the lack of the ability

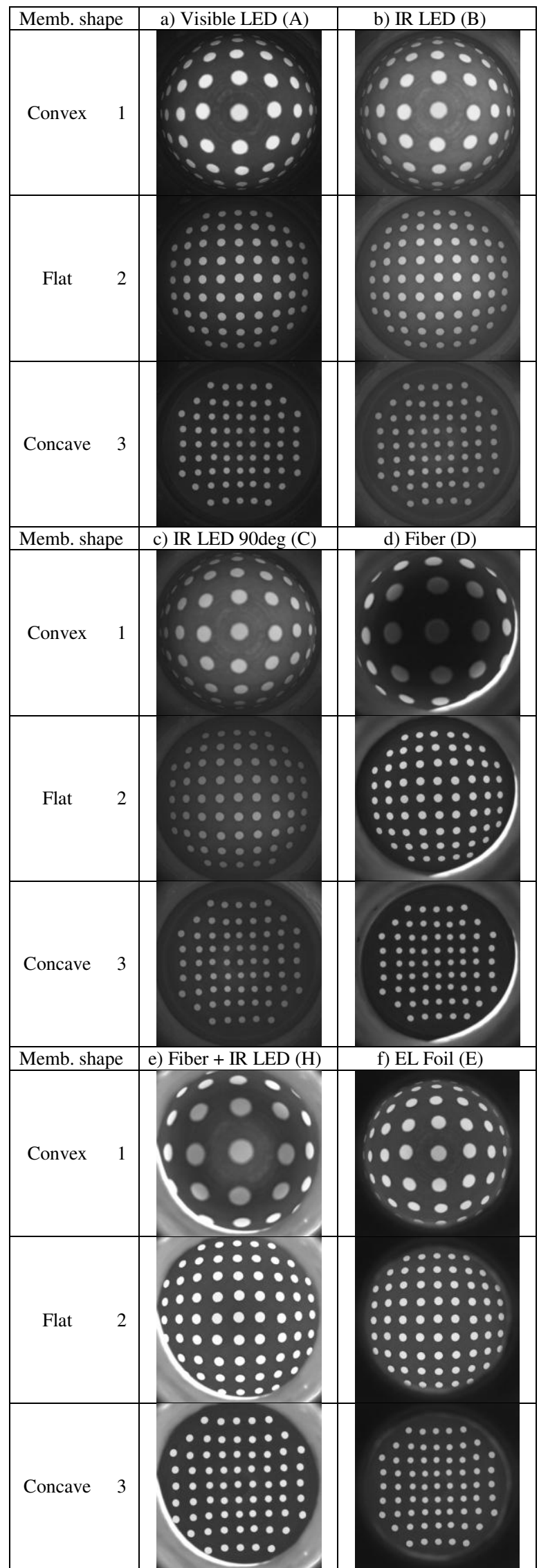

Fig. 12. Views of the studied model membranes, achieved at different lighting settings 
to arrange the optical fiber on the entire surface of the dome of the pneumatic chamber. This inconvenience was solved by creating a hybrid $(\mathrm{H})$ illuminator from systems $(\mathrm{C})$ and (D). In the produced system, the optical fiber illuminated the concave membrane and flat membrane, Figs. 12-3e and Fig. 12-2e, and illuminator (C) illuminated the convex membrane, Fig. 12-1e. Moreover, the fiber optic liner arranged on the top of the pneumatic dome dispersed the infrared light, and thus eliminated the spots previously observed on the membrane surface in system (C). Better results were obtained only when testing the illuminator based on electroluminescent film (E). In this case, all the membranes tested were illuminated properly, Fig. 12f.

The evaluation of lighting was done using the technique presented in point IV, and discussed in detail in $[5,19]$. Illuminators were examined on a group of three reference membranes shown in Fig. 10 (left). For all tested combinations of illuminator-membrane, measurement marker positions detection was conducted, the results of which are shown in Fig. 13. On this basis, the nodal points of polygons that reflect the surfaces of the tested membranes were determined using the technique presented in $[5,19]$. In the following step, by using the two-dimensional data interpolation method, the obtained nets were supplemented with nodes (compacted) to the required measurement resolution. Next, the degree of marker identification, Fig. 13, and the correctness of the shape of the generated grids were analyzed, Fig. 14.

\section{IMAGE PRE \& POST PROCESSING}

The tests were carried out using a fixed camera equipped with a wide angle lens $(\varphi=170 \mathrm{deg})$ with a fixed focal length $(F=1.2)$. The acquired images were subjected to a distortion compensation procedure developed by the authors $[8,9,23]$. As a result, the membrane views shown in Fig. 12 were obtained. These views show measuring markers with a diameter of $3 \mathrm{~mm}$ distributed on the surface of the reference membranes. The observed variations in the size of the spots in the images result from the marker approaching or moving away to/from the plane of the camera image sensor. This effect is further enhanced by the marker zooming out or approaching to/from the focus point. As a result, the view of the marker is blurred. This fact was used in [10] to measure the distance, and in [5] and [23] to determine the shape of the membrane surface in virtual and real space. The condition for determining the shape of the membrane surface is to perform image defuzzification. It was carried out using a thresholding operation that is always carried out with a threshold preset. As a result, a binary image is obtained, ready for labeling. During labeling, ( $\mathrm{x}, \mathrm{y})$ coordinates of the center of gravity for each spot, Fig. 13, as well as its area are determined. Knowledge of the determined area after the calibration of the measurement system is equivalent with knowing the distance from the marker to the camera image sensor [10]. During further analysis, only nodal points of the grid are processed

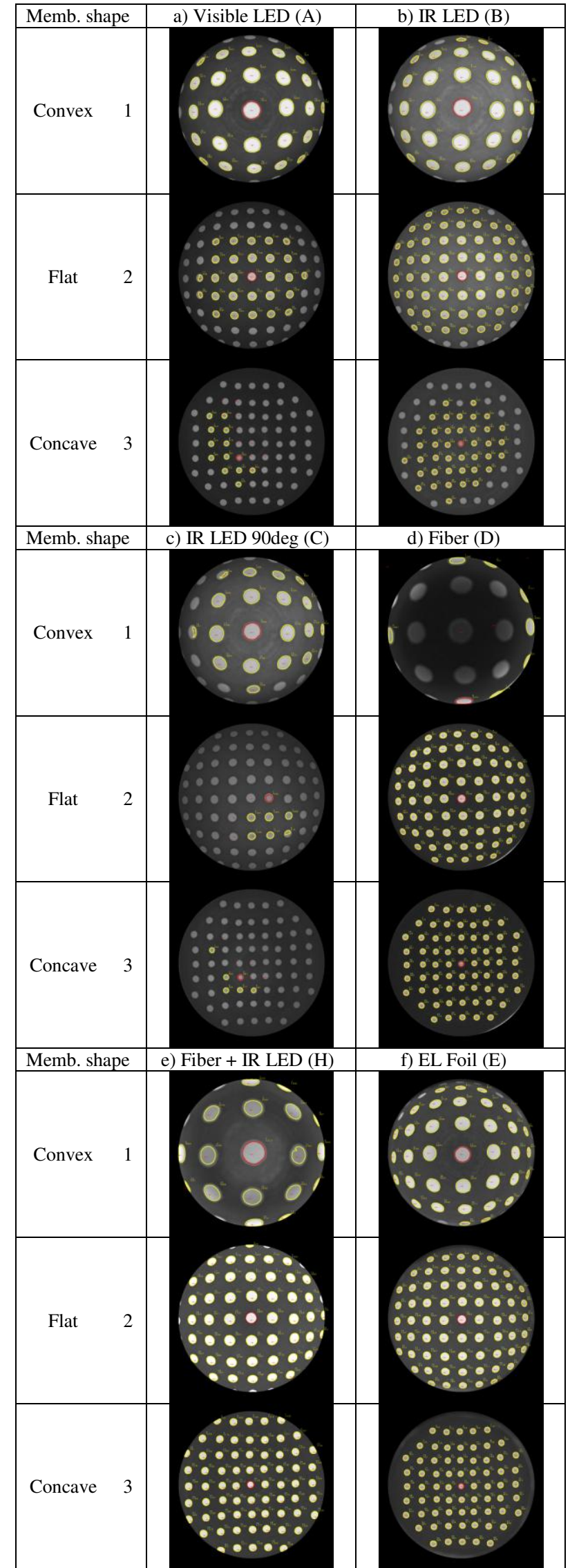

Fig. 13 Results of detecting the position of measurement markers achieved at different lighting settings 


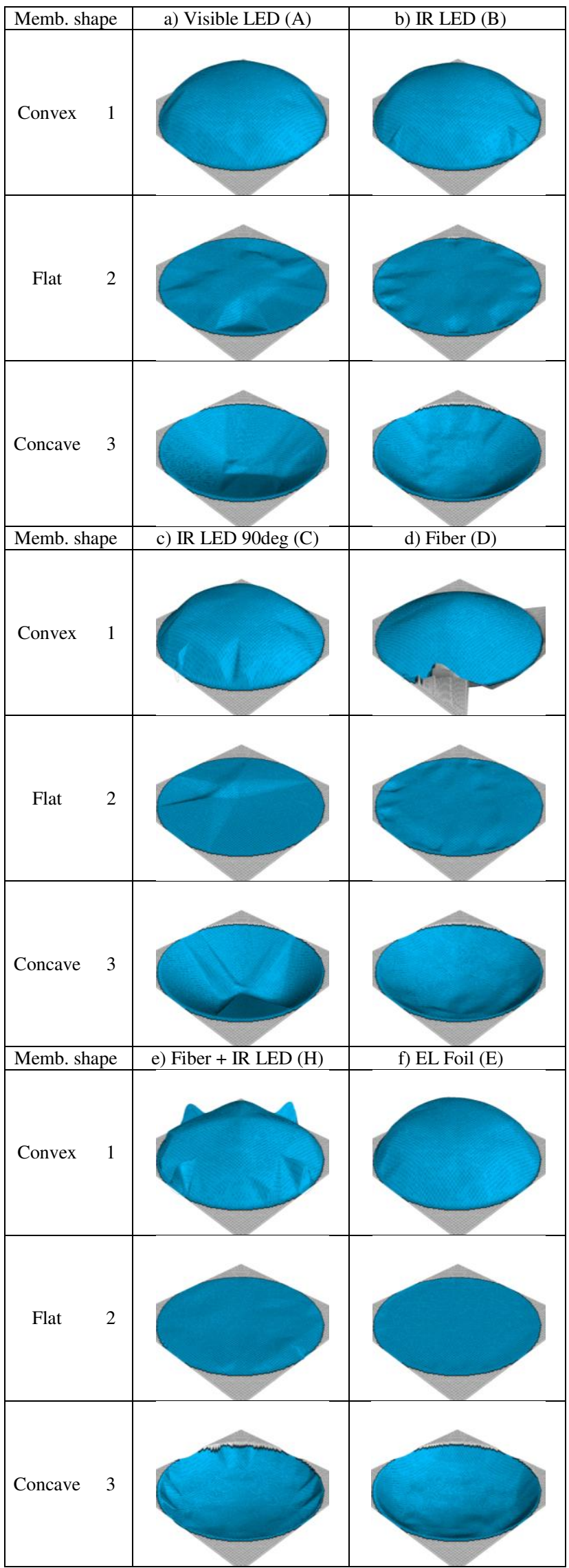

Fig. 14 Results of reconstructing the surface shape of the studied membranes achieved at different light settings with coordinates $\left(x_{i}, y_{i}, z_{i}\right)$ where $\mathrm{i}=1, \ldots, 69$. These points, using the interpolation method, determine a measuring grid of $300 \times 300$ points.

A graphical representation of the determined grids for all the studied membranes is presented in Fig. 14.

\section{RESULTS ANALYSIS}

The analysis of the obtained reconstructions of convex membrane shapes shows that the best shape mapping was obtained for illuminator (E). Next were solutions (A), (B), (C), (H) and (D). In solution (A), some overexposure is visible, which led to local flattening of the determined membrane surface shape. The case of solution (B) is similar with the difference that the entire membrane was illuminated except for the four markers that remained underexposed. In effect the distortion correction function and their values have been "improved", which is visible in the form of characteristic inequalities. When using illuminator (C), it was noticed that the power of light was not fully sufficient. As a result, some of the markers did not work at all, and several markers were underexposed. A result similar to solution (B) is observed for illuminator $(\mathrm{H})$. However, in this case the markers distant from the center of the membrane were overexposed and the markers located closer to the center were illuminated, but their brightness was noticeably lower. In addition, four markers under the given lighting conditions had too small a surface and were "improved" by the correction function. The worst illuminator in the study of the convex membranes was illuminator (D). Analyzing the picture shown in Fig. 13-1d it is easy to see that this state results from the underexposure of the middle portion of the membrane. No markers have been identified in this area. The obtained result is therefore the result of interpolation made on the basis of knowing the values of the characteristics determined for markers located only on the edge of the membrane.

The flat membrane, just like the convex membrane, was best visualized when illuminated with illuminator (E). A very good result was also obtained for illuminators $(\mathrm{H})$, (D) and (B). When using the illuminators (H) and (D), all the markers on the membrane were working, Fig. 13-2e and Fig. 13-2d. In solution (B) only seven markers less worked, Fig. 13-2b. In this group, the smallest distortion of the shape of the membrane was observed for illuminator $(\mathrm{H})$. Next came illuminator (D), and finally (B). When illuminating the membrane with system (D) the membrane was gently raised in the center. The use of the construction (B) allowed illuminating the central part of the membrane evenly, but the markers at the edge of the membrane were less illuminated. This is visible in the form of fluctuation observed near the edge of the membrane, Fig. 14-2b. The remaining solutions (A) and (C) did not work at all. When illuminator (A) was used only 22 markers placed in the center of the membrane were correctly marked. Along with them were still six markers operating, but they were not well-lit and, as a result, they were the cause of errors. In the case of illuminator (C), 
only five markers worked correctly, which could not lead to a correct membrane shape reconstruction.

The last test was to determine the shape of the concave membrane. The best results were obtained for illuminators (E), (D), (H) and (B). Illuminators (A) and (C) due to bad lighting of the membrane in its lower position, did not allow obtaining correct reconstruction results.

According to the authors, the shape of the concave membrane was best determined using illuminator (E). Then the sides of the membrane are reproduced correctly, and only minor remarks can be made to the shape of the central part of the membrane. In the second position, it was decided to place illuminator (D), which properly illuminated the measurement markers, and yet in the central part of the membrane reconstruction errors were noticeable. An equally good result was obtained for illuminator $(\mathrm{H})$. Just like in the previous case all markers on the obtained membrane shape "worked". As a result, the shape of the central part of the membrane is reproduced correctly (according to the authors even better than for illuminator (E)). However, disturbances were observed on the sides of the membrane, which forced illuminator $(\mathrm{H})$ to be classified in third position. The last discussed illuminator is construction (B). This system achieved a surprisingly good reconstruction result, taking into account that only 41 out of 69 markers worked properly. Their lack, however, explains the reconstruction errors observed on the side surfaces of the concave membrane.

\section{CONCLUSIONS}

The work discusses new illuminator constructions made for a vision sensor to measure the instantaneous stroke volume and the cardiac output of the heart pump. The experiments were carried out on a group of three reference membranes with a known mathematical description. Five variants of illuminators were subject to examination. Three tested constructions worked in the visible spectrum. The other two were implemented in the near-infrared band.

The experiments carried out have shown that it is possible to construct an illuminator that will effectively eliminate the problem of uneven surface illumination of a flaccid membrane. Among the illuminators, which design is partly based on LED diodes, the highest potential was demonstrated by the hybrid illuminator $(\mathrm{H})$. It combines the advantages of a fiber optic illuminator operating in the visible band (D) and illuminator (C), made of IR LEDs illuminating the sides. Although this variant requires two supply circuits, it allowed obtaining the correct shape of a flat and a concave membrane. Initially, the incorrectly determined shape of the surface of the convex membrane was finally corrected, increasing the control current of VSMG 10850 diodes. This operation was carried out programmatically by changing the setting of the PWM circuits.
The best of the examined systems was an illuminator built with the use of an electroluminescent film. The membrane surface shapes obtained were the closest to model shapes. This solution, however, due to the significant voltage supply in a commercially produced a heart-assisting pump, requires a special approach for the production of a pneumatic chamber.

The final effect of reconstructing the shape of the membrane surface also depends on the number and position of the markers. The tests were carried out taking 49 markers, Figs. 8 and 69 markers, Fig. 10 - 13. A larger number of markers, when properly illuminated and unfolded, more precisely determines the shape of the membrane surface sought. It is worth noting that, in principle, the material from which the diaphragm is made may be any. The test membrane was made of a graphite-silicone composite. It was used in the VAD, for which the SV was determined. The obtained values of the volume were affected by an error smaller than $4.8 \mathrm{ml}$, which constituted about $4 \%$ of the volume of the blood chamber VAD [23].

\section{AUTHOR'S STATEMENT}

The work presented in this publication was financed only by the authors' own funds.

\section{REFERENCES}

[1] Grad, L., Murawski, K., Pustelny, T., "Measuring the Stroke Volume of the Pneumatic Heart Prosthesis using an Artificial Neural Network", Proc. SPIE 10034, (2016), doi: 10.1117/12.2243952.

[2] Grad, L., Murawski, K., Sulej, W., "Research to Improve the Accuracy of Determining the Stroke Volume of an Artificial Ventricle Using the Wavelet Transform", Proc. SPIE 10455, (2017), doi: 10.1117/12.2280804.

[3] Murawski, K., Pustelny, T., Grad, L., Murawska, M., "Estimation of the Blood Volume in Pneumatically Controlled Ventricular Assist Device by Vision Sensor and Image Processing Technique", Proc. MMAR, 100 - 106, (2016), doi: 10.1109/MMAR.2016.7575115.

[4] Sulej, W., Murawski, K., "Determining the Stroke Volume of the Artificial Ventricle Using the Numerical Integration Method", Proc. SPA, 207 - 2012, (2017), doi: 10.23919/SPA.2017.8166865.

[5] Murawski, K. Murawska, M., Pustelny, T., "The System and Method of Determining the Shape of the Membrane Pneumatic Pump of Extracorporeal Heart Assist Device", Patent Application: nr P.414104, 2015. (in Polish).

[6] Kustosz, R., Jarosz, A., Gawlikowski, M., Kapis, A., Gonsior, M., "The Role and Perspectives of Development of the Polish Air Pump Heart Assist on the Market of Heart Prosthetic", Polish artificial heart, the development of design, qualification tests, preclinical and clinical, ISBN 978-83-63310-12-7, 2013, (in Polish).

[7] Sulej, W., Murawski, K., "The Membrane Shape Mapping of the Artificial Ventricle in the Actual Dimensions", Proc. FEDCSIS, $675-$ 680, (2017), doi: 10.15439/2017F269.

[8] Sulej, W., Murawski, K., Pustelny, T., "Improvement of Accuracy of the Membrane Shape Mapping of the Artificial Ventricle by Eliminating Optical Distortion”, Proc. MMAR, 799 - 804, (2017), doi: 10.1109/MMAR.2017.8046934.

[9] Sulej, W.; Murawski, K.; Pustelny, T. "Optical Distortion Compensation in Visual Measurement with a New Depth From Defocus Method", Photonics Letters of Poland, 9, 4, 122 - 124, (2017), doi: 10.4302/plpv9i4.775.

[10] Murawski, K., "Method of Measuring the Distance using One Camera", Patent Application: P.408076, 2014. (in Polish).

[11] Murawski, K., "Method of Measuring the Distance to an Object Based on One Shot Obtained from a Motionless Camera with a Fixed-Focus 
Lens”, Acta Physica Polonica A 127 (6), 1591-1595 (2015). DOI: 10.12693/APhysPolA.127.1591

[12] Murawski, K., Arciuch, A., Pustelny, T., "A New Innovative Depth From Defocus Method - Identification of the Impact of the Marker Size on the Distance Measurement Result", Proc. SPIE 10034, (2016), doi: $10.1117 / 12.2244130$.

[13] Murawski, K., Arciuch, A., Pustelny, T., "Studying the Influence of Object Size on the Range of Distance Measurement in the new Depth From Defocus Method", Proc. FEDCSIS, 817 - 822, (2016), doi:10.15439/2016F136

[14] Murawski, K.; Arciuch, A.; Pustelny, T. "Study of Distance Range Visual Measurement in the new Depth From Defocus Method",Photonics Letters of Poland, 8, 2, 48 - 50, (2016), doi:10.4302/plp.2016.2.07.

[15] Murawski, K., "Measurement of Membrane Displacement with Motionless Camera Equipped with a Fixed Focus Lens", Metrology And Measurement Systems, 22, 1, 69 - 78, (2015), doi: 10.1515/mms2015-0011.

[16] Murawski, K., "Measurement of Membrane Displacement Using a
Motionless Camera", Acta Physica Polonica A 128 (1), 10-14, (2015). doi: 10.12693/APhysPolA.128.10.

[17] Murawski, K., "New Vision Sensor to Measure and Monitor Gas Pressure", Acta Physica Polonica A 128 (1), 6 - 9, (2015). doi: 10.12693/APhysPolA.128.6.

[18] Murawski, K., "New Vision Sensor to Measure Gas Pressure", Measurement Science Review, 15, 3, 132 - 138, (2015), doi: 10.1515/ msr-2015-0020.

[19] Sulej, W., Grad, L., Murawski, K., "The Technique of Accuracy Measurement of Membrane Shape Mapping of an Artificial Ventricle", Proc. SPIE 10455, (2017), doi: 10.1117/12.2280806.

[20] https://www.nxp.com/docs/en/data-sheet/PCA9622.pdf.

[21] http://www.soled.pl/oswietlenie-swiatlowodowe/swiatlowodyswiecace-bokiem.html

[22] http://www.microchip.com

[23] Sulej, W., "Measurement of the stroke volume of the extracorporeal pneumatic heart assist pump using image processing and analysis techniques", PhD. Dissertation, Military University of Technology, Warsaw, (2018) - in Polish. 